\title{
A UTILIZAÇÃO DE FERRAMENTAS DE MONITORAMENTO PARA A OTIMIZAÇÃO DO GERENCIAMENTO DA REDE
}

\author{
THE USE OF MONITORING TOOLS FOR NETWORK MANAGEMENT \\ OPTIMIZATION
}

\author{
Luís Carlos Belentani - lcbele@ yahoo.com.br \\ Juliano Marcello - jmarcello@uniara.com.br \\ Fabiana Florian - fflorian@ uniara.com.br \\ Universidade de Araraquara (UNIARA) - Araraquara - SP - Brasil
}

DOI: 10.31510/infa.v15i2.509

\begin{abstract}
RESUMO
A crescente disseminação de redes de computadores associado a integração densa de componentes eletrônicos e o elevado número de ferramentas para o auxílio de gestão têm-se a necessidade de um gerenciamento eficaz destas redes complexas, desta forma este trabalho evidencia a importância de algumas ferramentas (NAGIOS, PRTG E ZABBIX). Desta maneira, o foco deste trabalho consiste-se em despertar nos gestores de rede a busca por mecanismo de otimização; para seus respectivos gerenciamentos da rede, por isso o objetivo deste trabalho constitui-se em garantir novos conhecimentos a respeito de gerenciamento e monitoramento de redes, assim como a relevância da utilização de ferramentas que facilitem as atividades diárias do profissional de gestão de Tecnologia da Informação (TI). Diante disso, o trabalho perpassa o entendimento das estruturas das ferramentas de monitoramento Zabbix, Nágios e PRTG e como estas utilizam dados para elaboração de notas sobre as informações coletadas na rede.
\end{abstract}

Palavras-Chave: Gerenciamento da Rede. Ferramentas de Monitoramento de Rede. Otimização da Rede.

\begin{abstract}
The increasing dissemination of computer networks associated with the dense integration of electronic components and the high number of tools for the management aid have the need of an effective management of these complex networks, in this way this work evidences the importance of some tools (NAGIOS , PRTG and ZABBIX). In this way, the focus of this work consists of awakening in the network managers the search for optimization mechanism; for their respective management of the network, so the objective of this work is to guarantee new knowledge regarding management and monitoring of networks, as well as the relevance of the use of tools that facilitate the daily activities of the professional of Information Technology management (YOU). Therefore, the work goes through the understanding of the structures of Zabbix, Nagios and PRTG monitoring tools, and how they use data to prepare notes on the information collected in the network.
\end{abstract}


Keywords: Network Management. Network Monitoring Tools. Network Optimization.

\section{INTRODUÇÃO}

A multiplicação de usuários da Internet como ferramenta indispensável do cotidiano fez com que a necessidade de que os sistemas fornecidos pelas redes de computadores evoluíssem a cada ano e, assim, impulsionassem o desenvolvimento tanto das redes internas (intranet) quanto externas (internet).

Neste contexto, surgiu a necessidade de gerenciamento e monitoramento de redes com a finalidade de garantir a disponibilidade de serviços em perfeito funcionamento, por meio de coleta de dados dos instrumentos gerenciados. O monitoramento exibe, com detalhes, estatísticas sobre o desempenho e performance sobre o funcionamento dos dispositivos, utilizando-se de dados anteriores sobre o que foge à normalidade, com o objetivo de corrigir o erro antes de um possível comprometimento da rede.

É importante ressaltar que a propagação de redes de dimensões diferenciadas e a adaptação com uma infinidade de componentes eletrônicos, como smartphones, Smart TV, entre outros, torna a utilização destas redes ainda mais complexas; vindo a, crescer a oferta de vários mecanismos, com suas vantagens e desvantagens.

Por isso, a imprescindibilidade de supervisão destas redes ante o crescimento tecnológico requer ainda o desenvolvimento de programas mais sofisticados tecnicamente, e que abarquem uma série de características adaptadas à expansão de novas tecnologias. Assim, o gerenciamento de tais redes tornou-se uma tarefa indispensável para manter o seu funcionamento correto (STALLINGS, 1998).

Assim, nota-se que o monitoramento da rede permite controlar seus recursos, além de identificar e prevenir danos. O trabalho de gerenciamento e monitoramento de redes envolve alguns etapas, tais como: configuração, performance, falhas e segurança.

Desta maneira, este artigo apresenta uma análise sobre algumas ferramentas (Zabbix, Nagios e PRTG) de gestão e monitoramento de redes capazes de demonstrar informações sobre o acompanhamento dos ativos pertencentes à rede.

Este trabalho tem por objetivo - garantir novos conhecimentos sobre gerenciamento e monitoramento de redes, assim como a relevância da utilização do uso de ferramentas que facilitem as atividades diárias do profissional de gestão de Tecnologia da Informação (TI). Temse, ainda, a pretensão de entender as estruturas das ferramentas de monitoramento Zabbix, 
Nagios e PRTG e como as utilizam dados para elaboração de notas sobre as informações coletadas na rede.

Há no mercado uma série de ferramentas disponíveis para gestão de rede, no entanto, é necessário ter conhecimento técnico conceitual sobre a adequada utilização delas. Este trabalho se justifica ao buscar mecanismos que otimizem o gerenciamento de redes, ou seja, que sejam capazes de ser eficientes e eficazes para prevenção de problemas.

Em um procedimento operacional da gestão da rede, as ferramentas vêm agregar ao processo de trabalho com que os softwares principais, neste nicho, podem ofertar.

\section{PRINCIPAIS TIPOS DE TECNOLOGIAS DE ACESSO À INTERNET}

Atualmente, existem diversos tipos de tecnologias empregadas para o acesso do usuário à internet, sejam elas sem fio, satélite, fibra, $\mathrm{DSL}^{1}$, dentre outros. Há muitas tecnologias que são ofertadas pelas empresas mas, de modo geral, estas empresas não informam como os provedores de internet utilizam suas redes, e desta forma, se faz necessário compreender o lado tecnológico que envolve as redes e suas complexidades.

Assim, um tipo de tecnologia é a fibra óptica, segundo Kurose e Ross Apudi Scapin (2015), o sinal é leve e o meio é um tipo especial de vidro flexível ou cabo de plástico transparente, assim sendo, o vidro permite que a luz viaje muito bem, ainda de acordo com os mesmos autores, em uma explicação simplista de como funciona um sistema de comunicação por fibra óptica: existe um transmissor em uma extremidade que converte o sinal elétrico em luz, assim, ele pulsa de maneira semelhante a como o Código Morse pulsa. A luz percorre no cabo de vidro até um receptor na outra extremidade e o receptor detecta a luz que gera um sinal elétrico no computador do usuário.

Cabe ressaltar que a luz, viaja mais rápido que a eletricidade, pelo menos quando se trata de eletricidade que flui através de um fio de cobre. Conforme a Science Line da UCSB: " $A$ luz viaja pelo espaço vazio a 186.000 milhas por segundo. A eletricidade que flui através dos fios em suas casas e aparelhos viaja:apenas cerca de 1/100 da velocidade da luz."

Outra modalidade de acesso a internet é a conexão sem fio, o termo "sem fio" é um conceito bastante abrangente que abarca qualquer tipo de acesso à Internet que não exija um

${ }^{1}$ Digital Subscriber Line 
cabo entre usuário e seu ISP ${ }^{2}$, portanto, isso faz com que seja importante compreender como cada tipo funciona, bem como suas vantagens e desvantagens, como por exemplo a banda larga sem fio, a internet móvel e a internet via satélite.

Banda larga sem fio é quando os ISPs anunciam banda larga sem fio para o local de acesso do computador do usuário, o ISP se conectará à Internet por meio de uma conexão por cabo e transmitirá essa conexão usando ondas de rádio.

Outra modalidade de conexão sem fio, segundo Battisti (2007), é a internet móvel, ainda segundo o mesmo autor, é assim que o usuário obtém a Internet em seu telefone, pen drive ou placas de PC que entram no seu computador. Os provedores de serviços geralmente se referem a ele como Banda Larga Sem Fio Móvel, embora o termo banda larga não esteja sendo usado tecnicamente corretamente.

A Internet móvel funciona com ondas de rádio, semelhantes à banda larga sem fio. A maioria das pessoas não percebe que seus celulares são, na verdade, um tipo de rádio. Ao longo dos anos, os provedores de serviços descobriram uma maneira de transmitir voz e dados ao mesmo tempo. Existem várias maneiras diferentes que os dados podem ser enviados através do sinal da célula.

Outra modalidade de conexão é a internet via satélite, de acordo com Battisti (2007), como o nome sugere, esta é uma maneira de obter acesso à Internet através de uma antena parabólica. O sinal é transmitido para um satélite e este envia o sinal para o usuário e viceversa. Como a banda larga sem fio, é uma tecnologia de linha de visão. Seu prato precisa ter uma visão clara de onde quer que o satélite esteja no céu.

\subsection{Monitoramento da Rede}

De acordo com Kurose e Ross Apudi Scapin (2015), para qualquer nova ferramenta de monitoramento e gerenciamento de rede, a primeira etapa é avaliar seu inventário existente. Desta forma, permite-se que a ferramenta descubra todos os seus dispositivos. O software pode alertar o usuário sobre informações que nem ele mesmo sabia que tinha. O monitoramento de rede e o gerenciamento de ferramentas podem ser inteligentes o suficiente para propor maneiras de melhorar seu sistema: ele pode sugerir novas configurações ou destacar gargalos. A última etapa é deixar o $\mathrm{SNMP}^{3}$ interromper o fluxo e informá-lo sobre o uso diário da rede.

\footnotetext{
${ }^{2}$ Internet Service Provider

${ }^{3}$ Simple Network Management Protocol
} 
Há cinco as áreas funcionais no gerenciamento num ambiente OSI, de acordo com Battisti (2007): Gerência de configuração; Gerência de desempenho; Gerência de falhas; Gerência de contabilidade; Gerência de segurança.

Uma vantagem do modelo OSI, que é um sistema baseado na orientação a objetos, de acordo com Battisti (2007), é que ele permitiu a inovação nas camadas individuais. Essas camadas são abstraídas umas das outras, o que impede a emprego acidental de dependências em outras camadas.

Embora o layout da rede e os fluxos de pacotes sejam importantes, torna-se limitante fazê-lo sem o contexto dos aplicativos executados na parte superior da rede. É como tentar analisar seu consumo de energia observando um diagrama de fiação elétrica em sua casa, sem considerar os dispositivos conectados a ele, sem examinar as tensões que os aplicativos colocam em uma rede na Camada, o usuário tem uma imagem incompleta das demais Camadas. $\mathrm{O}$ gerenciamento de aplicativos oferece um ângulo melhor e esses serviços estão em alta demanda, de acordo com Saito ${ }^{7}$ (2001), e possível evidenciar o funcionamneto dos sistemas.

Segundo Saito $^{7}$ (2001), os desenvolvedores, de modo geral, preferem essa abordagem porque não há criação de dependência. Os sistemas baseados em agentes desviam os recursos dos principais componentes de um aplicativo.

Saito $^{4}$ (2001) ainda acrescente que este é um diagrama de arquitetura de aplicativo que mostra as interações entre os diferentes componentes. Os diagramas gerados não conterão roteadores, switches e outros componentes de rede invisíveis. Diagramas de rede podem ser difíceis de manter manualmente, da mesma forma que os diagramas de aplicativos são igualmente difíceis. Essa automação não apenas facilita a vida do desenvolvedor, como também fornece um mapa dentro de cada rede e os engenheiros de aplicativos podem ter conversas construtivas.

Pode-se compreender que, no Rastreamento transacional, os processos são simplificados com um diagrama de arquitetura de aplicativo baseado nas abordagens de coleta de dados, assim "As plataformas de monitoramento e gerenciamento de aplicativos poderão usar esse diagrama para mostrar o fluxo transacional individual e agregado entre os componentes. É aqui que a combinação de ferramentas de aplicativos e de rede é sinergizada (MICROSOFT, 2018).

\footnotetext{
4 DIAGRAMA de arquitetura. 2018. Disponível em: <https://msdn.microsoft.com/ptbr/library/hh351451(v=azure.10).aspx>. Acesso em: 13 set. 2018.
} 
Diante disso, é importante ressaltar, que as ferramentas clássicas de gerenciamento e monitoramento de rede continuam sendo vitais, mas usá-las exclusivamente coloca a organização usuária em uma espécie de vácuo, segundo Battisti (2007). Em vez disso, o uso de ferramentas de monitoramento e gerenciamento de rede e aplicativos otimizam os processos, desta maneira:

O gerenciamento de rede pode ser visto como um conjunto de mecanismos operacionais e administrativos necessários para controlar os recursos da rede, manter os recursos da rede operacionais, facilitar o aumento da rede, gerenciar os recursos e controlar o acesso à rede (SCAPIN, 2015, p.15).

Já para Kurose e Ross Apudi Scapin (2015, p.15) “o Gerenciamento de rede inclui a disponibilização, a integração e a coordenação de elementos de hardware, software $e$ humanos, para monitorar, testar, consultar, configurar, analisar, avaliar e controlar os recursos da rede, e de elementos", a fim de atender todas as demandas.

Além disso, afirma Scapin (2015) que o “ monitoramento e gestão facilitam que o administrador da rede tenha uma visão mais precisa, em tempo real, do que acontece na rede", ou seja, identifica precocemente os problemas que poderão causar os danos posteriormente, e assim evitar prejuízos tanto para os clientes quanto para o empreendedor.

O objetivo da Gerência de Redes é monitorar e controlar os elementos da rede (sejam eles físicos ou lógicos), assegurando um certo nível de qualidade de serviço. Para realizar esta tarefa, os gerentes de redes são gera lmente auxiliados por um sistema de gerência de redes (LOPES Apudi SCAPIN, 2015, p.15).

Além disso, Scapin (2015); leciona que "no desenvolvimento modelo OSI (Open Systems Connection) foram definidas cinco áreas do gerenciamento: Falhas, Configuração, Contabilidade, Desempenho e Segurança”, originando a sigla em Língua Portuguesa (FCCDS) ou Língua Inglesa (FCAPS).

\subsection{FCAPS $^{5}$}

Este modelo, possui como foco principal o gerenciamento, conforme Scapin (2015):

\section{Quadro 1 - FCAPS}

\footnotetext{
5 Faults (falhas), Configuration (configuração), Accounting (contabilidade), Performance (Desempenho) e Security (segurança)
} 
- Faults $^{9} \rightarrow$ Esta focalizado em identificar as falhas diagnosticando suas respectivas origens, mantendo-as isoladas da rede, reparando-as.

- Configuration $^{9} \rightarrow$ supervisiona e monitora os estados do ambiente de rede, mantendo tanto os alistamentos de dispositivos de rede e também a documentação das deformações físicas e lógicas da rede.

- Accounting ${ }^{9} \rightarrow$ consiste-se no gerenciamento contábil, viabilizando a mensuração dos custos de uso da rede. Ainda possibilita o crescimento da rede e identifica abusos na utilização dos recursos.

- Performance ${ }^{9} \rightarrow$ oferece ferramentas de monitoramento de desempenho, modificação e controle da utilização de recursos, viabilizando o tráfego mínimo para a rede.

- Security ${ }^{9} \rightarrow$ mantém a segurança de informações e plenitude dos dados, estando ligada à autenticação e permissão de usuários.

Fonte: SCAPIN, (2015).

\subsection{Protocolo SNMP6}

Para Battisti (2007), o Protocolo SNMP tem como função simplificar a troca de dados de monitoramento entre os dispositivos da rede. Para tanto, disponibiliza dados de status dos elementos da rede em atividade e estatísticas significativas para seu andamento.

\footnotetext{
consiste-se num os protocolos mais utilizados, ao longo dos anos, até chegar à versão mais recente, SNMPv3 que utiliza o protocolo UDP (User Datagram Protocol) com o objetivo de facilitar a comunicação entre as NMS (Network Management Systems) e os dispositivos gerenciados. O SNMP é encarregado de fornecer dados como percentual de utilização do disco rígido, status do funcionamento da rede (SCAPIN, 2015, p.17).
}

Assim, o monitoramento de rede, efetuado por este protocolo SNMP, conforme Scapin (2015) constitui de três itens: 
a) os Dispositivos Gerenciados - que coleta e armazena dados para que possam ser usados pelas NMSs, podendo ser diversos elementos da rede tais como: como os Servidores, roteadores, switches, etc;

b) os Agentes ou indicadores do programa - que reúnem dados sobre os dispositivos regidos em MIBs, dados como uso de memória, processamento e outros;

c) NMS, sistemas de gerenciamento de redes - encarregados de monitorar e controlar os dispositivos. Tem como opção a visualização gráfica dos dados coletados e armazenados nas MIBs (Management Information Base). Assim, acrescenta Scapin (2015) "os sistemas de gerenciamento de redes são responsáveis pelo monitoramento e controle dos dispositivos e trazem opções de visualizar graficamente as informações coletadas e armazenadas nas MIBs".

Fonte: SCAPIN, (2015).

$\mathbf{M I B}^{7}$ - que se consiste na "base de dados onde são depositadas as informações colhidas pela máquina administradora, localizada nas próprias máquinas da rede” (Scapin, 2015), assim percebe-se que esta base de dados constitui um dos elementos mais importante neste processo de coleta e armazenamento de informações.

\subsection{Sistemas de Gestão e Monitoramento de Redes}

Scapin (2015) afirma que "tem-se buscado soluções em gestão de redes de caráter preventivo e também mais eficazes, através de programas de monitoração e gerenciamento ainda mais proativos", desta forma infere-se que existe um "leque de possibilidades" ou ferramentas para auxiliar no gerenciamento de redes.

Assim, percebe-se que tais acessórios possuem como finalidade principal, assegurar e possibilitar o desempenho conveniente das redes, em qualquer estabelecimento de pequeno, médio e grande porte, apresentando como soluções, três ferramentas: Nagios, Zabbix e PRTG.

$\rightarrow$ Nágios - Tendo como criador e idealizador, Ethan Galstald, inicialmente rodava apenas no Linux, constitui uma ferramenta de monitoramento de redes open-source, coletando dados sobre as funções possuindo como meta principal gerar dados estatísticos que possibilitem a melhoria no desempenho desta rede. Esta ferramenta, atualmente pode ser executada nos

\footnotetext{
${ }^{7}$ Management Information Base
} 
seguintes sistemas conforme Scapin (2015): “Unix/Linux, no Windows, pelo programa Nag Win e no Mac OS/X. Existem pacotes também para Fedora, Ubuntu, SUSE e Debian”.

$\rightarrow$ PRTG Network Monitor - PRTG Network Monitor é um instrumento de monitoramento, fundamentado na plataforma operacional Windows. É considerado apropriado tanto para redes de pequeno porte quanto para redes de médio e grande porte. Na busca de informações para monitoramento, o PRTG utiliza-se de muitos protocolos de Gerência de Rede conhecidos, dentre eles destacam-se: Simple Network Management Protocol (SNMP), Windows Management Instrumentation (WMI), packet sniffer, Cisco Net Flow (assim como sFlow e jFlow) e muitos outros. As informações aqui coletadas, em sua totalidade, são guardadas em um banco de dados interno possibilitando uma visão de longo prazo sobre o estado geral e sobre a utilização da rede.

$\rightarrow$ Zabbix - consiste-se numa "solução de gerenciamento de redes de todos os portes distribuído sob a licença GPL. Ao cobrir defeitos dos concorrentes, que obrigava os administradores a usarem mais de duas ferramentas ao mesmo tempo" (ZABBIX Apudi SCAPIN, 2018).

\section{PROCEDIMENTOS METODOLÓGICOS}

Sabe-se que numa pesquisa, uma das fases mais importantes da mesma, consiste-se na escolha da estratégia de investigação, a fim de que defenda plenamente a tese defendida pelo pesquisador.

Desta forma, este trabalho foi desenvolvido por meio do levantamento bibliográfico, através da consulta a revistas e portais eletrônicos, livros ou ainda teses e artigos científicos, objetivando encontrar referencial teórico de autores referência no assunto.

Além disso, este artigo, foi desenvolvido a partir do estudo de caso. Estudo de caso, empiricamente pode ser definido como uma proposta de avaliação e resolução de uma situação problema.

Assim, para Meirinhos e Osório (2010) o “método é experimental (hipotéticodedutivo) e o conhecimento extraído da realidade natural ou social é estável e quantificável, a partir de um distanciamento entre o investigador e a realidade estudada”. 


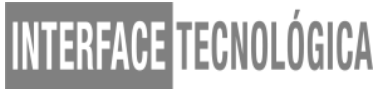

No entanto, Freitas e Jabbour (2011) afirmam que "quando a finalidade da investigação é descritiva ou causal, a abordagem é quantitativa; quando a finalidade é explicar ou descrever um evento ou uma situação, a abordagem adotada deve ser a qualitativa".

Assim, este artigo possui grande relevância científica e acadêmica, uma vez que o mesmo poderá ser usado como referencial teórico de outros pesquisadores.

\section{RESULTADOS E DISCUSSÃO}

As vantagens do gerenciamento de redes são muitas, cabe ressaltar que o gerenciamento de redes gera uma otimização dos custos de instalação de quaisquer dispositivos, minimização dos esforços relacionados à engenharia e à manutenção da rede. Além das vantagens físicas, são notadas também, dentro do gerenciamento de redes, várias outras vantagens, como a operacional, ao reduzir o número de chamados.

Assim, faz-se necessário identificar e mensurar o número de chamados que esse problema gera, para, assim, poder evidenciar os aspectos positivos de um gerenciamento de rede.

\subsection{Atenuação de Sinal Óptico}

A atenuação ou perda de transmissão pode ser definida como a diminuição da intensidade de energia de um sinal ao propagar-se através de um meio de transmissão. Nas fibras ópticas, a atenuação varia de acordo com o comprimento de onda da luz utilizada, essa atenuação é a soma de várias perdas ligadas ao material que é empregado na fabricação das fibras e à estrutura do guia de onda. O que pode provocar a atenuação pode ser absorção, espalhamento ou deformação mecânica, esse último item mencionado, foi o caso evidenciado neste artigo. A empresa faz a rotina mensal de manutenção preventiva em seu Backbone, como checar e fazer limpeza no ar condicionado, verificar fusíveis de retificadoras e organizar cordão óptico no rack, e justamente na organização do cordão óptico o colaborador não tomou a precaução devida e na organização do cordão excedeu a curvatura máxima permitida, e assim atenuando o sinal óptico. 
Como a preventiva é feita de madrugada, e o trafego de rede é baixo, não houve perca de pacotes na porta do switch, mas com o decorrer do dia, houve aumento de trafego e o sinal atenuou, assim começou a ter perdas de pacotes nesta interface, mas neste momento o Zabbix identificou a perda de sinal e enviou o alerta para equipe de rede.

\section{CONSIDERAÇÕES FINAIS}

A multiplicação de usuários da Internet como ferramenta indispensável do cotidiano fez com que a necessidade de que os sistemas fornecidos pelas redes de computadores evoluíssem a cada ano e, assim, impulsionassem o desenvolvimento tanto das redes internas (intranet) quanto externas (internet).

Neste contexto, surgiu a necessidade de gerenciamento e monitoramento de redes com a finalidade de garantir a disponibilidade de serviços em perfeito funcionamento, por meio de coleta de dados dos instrumentos gerenciados. O monitoramento exibe, com detalhes, estatísticas sobre o desempenho e performance sobre o funcionamento dos dispositivos, utilizando-se de dados anteriores sobre o que foge à normalidade, com o objetivo de corrigir o erro antes de um possível comprometimento da rede.

O presente trabalho apresentou uma análise sobre algumas ferramentas de gestão e monitoramento de redes capazes de demonstrar informações sobre o andamento dos ativos pertencentes à rede, além de apresentar e comparar três ferramentas de gerenciamento e monitoramento de rede: Zabbix, Nagios e PRTG.

Sendo assim, esse trabalho tem por objetivo garantir novos conhecimentos sobre gerenciamento e monitoramento de redes, assim como a relevância da utilização do uso de ferramentas que facilitem o trabalho. Tem-se, ainda, a pretensão de entender as estruturas das ferramentas de monitoramento Zabbix, Nagios e PRTG e como utilizam dados para elaboração de notas sobre as informações coletadas na rede.

A justificativa do presente estudo deu-se, pela necessidade do mercado, atualmente, em buscar mecanismos de otimização de gerenciamento de redes e a questão-problema que gerou a busca das informações foi inclinada para a seguinte pergunta: Como são os processos de trabalho e como trabalham os principais instrumentos das ferramentas de monitoramento para a otimização do gerenciamento da rede e com o quê os softwares principais, neste nicho, podem ofertar. 


\section{REFERÊNCIAS}

AVALLE, R.P Gerenciamento de Redes. Disponível em: <www.gta.ufrj.br/gra/99/rodrigo/ger_redes.html.>, Acesso em: 18 Fev. 2018.

BATTISTI, Gerson. Modelo de Gerenciamento para Infraestruturas de Medições de Desempenho em Redes de Computadores. 2007. Disponível em: < https://lume.ufrgs.br/bitstream/handle/10183/12671/000632788.pdf?sequ>. Acesso em: 15 ago. 2018.

FREITAS, Wesley. R. S.; JABBOUR, Charbel J. C. Utilizando Estudo De Caso(S) Como Estratégia de Pesquisa Qualitativa: Boas Práticas e Sugestões. 2011. Disponível em: <https://www3.ufpe.br/moinhojuridico/images/ppgd/8.12a\%20estudo\%20de\%20caso.pdf >.

Acesso em: 28 jul. 2018.

MEIRINHOS, M.; OSÓRIO, A. O estudo de caso como estratégia de investigação em educação: Instituto Politécnico de Bragança. 2010. Disponível em: <https://bibliotecadigital.ipb.pt/bitstream/10198/3961/1/O\%20estudo\%20de\%20caso\%20com o\%20estrat\%C3\%A9gia\%20de\%20investiga\%C3\%A7\%C3\%A3o\%20em\%20educa\%C3\%A 7\%C3\%A3o.pdf>. Acesso em: 28 jul. 2018.

DIAGRAMA de arquitetura. 2018. Disponível em: <https://msdn.microsoft.com/ptbr/library/hh351451(v=azure.10).aspx>. Acesso em: 13 set. 2018.

SCAPIN, A. H. Análise de Ferramentas de Gerência de Redes e Interfaces Web. 2015. Disponível em: <http://www.redes.ufsm.br/docs/tccs/Alex-Scapin.pdf>. Acesso em: 15 ago. 2018.

STALLINGS, W. SNMP, SNMPv2, SNMPv3 and RMON 1 and 2: the practical guide to network management standards. 3rd ed. Reding: Addison-Wesley, 1999.http://www.paessler.com/knowledgebase/en/topic/41373-is-there-a-site-planner-toolforplanning-large-installations-of-prtg-network-monitor Acesso em: 16 Abr.2018. 